\title{
DENSITY TOPOLOGY INVOLVING MICROSCOPIC SETS AND CATEGORY
}

\author{
ElŻBIETA WAgneR-BoJaKOWsKa - WŁAdYsŁaW WilcZYŃSKI - \\ - WoJCIECH WoJdOWsKI \\ Dedicated to the memory \\ of Polish mathematicians: Marian Rejewski, Jerzy Różycki and Henryk Zygalski.
}

\begin{abstract}
Analogously to the method used for $c$-density point, we introduce the notion of $c_{\mu}$-density point for arbitrary set $A$ having the Baire property, replacing the covergence in measure with the convergence with respect to the $\sigma$-ideal of microscopic sets. Then, we consider the density type topology $\mathcal{T}_{c_{\mu}}$ generated by $c_{\mu}$-density operator and study basic properties of $\mathcal{T}_{c_{\mu}}$ in comparison with the classical density, $\mathcal{I}$-density and $c$-density topologies.
\end{abstract}

The notion of a density point has been studied and developed extensively since the notion of a density topology $\mathcal{T}_{d}$ was introduced by $\mathrm{H} \mathrm{a} \mathrm{u} \mathrm{p} \mathrm{t}$ and $\mathrm{P}$ a u c in 1952 [8]. It is interesting that the related notion of approximate continuity, as defined by Denjoy in 1915 [3, had been known far earlier and utilized in the study of the theory of integration. The properties of the density topology were discovered gradually by G of fman, Ne u gebauer and Nishiura [6], Goffman and Waterman [7] and Tall [16]. The theory seemed to be mostly complete in the late seventies (see [11). However, in 1981, the definition was reformulated without the use of the notion of measure (see [18]).

Let $\mathcal{B}$ denote a family of all sets having the Baire property and $\mathcal{I}$ the $\sigma$-ideal of sets of the first category. It is well-known (see [11. Theorem 4.6]) that any set $A$ having the Baire property can be represented in the form $A=G \triangle P$, where $G$ is regular open and $P$ is of the first category. Additionally, the representation is unique in any space in which every non-empty open set is of the second category, so it is unique on the real line. The regular open set is the biggest one which can be used in this representation. Further, $n \cdot A=\{n x: x \in A\}$, $A-x_{0}=\left\{x-x_{0}: x \in A\right\}, A \sim B$ means that $A \triangle B \in \mathcal{I}$ and $m(A)$ stands for the Lebesgue measure of a measurable set $A$. For $A \subset \mathbb{R}$, let $\bar{A}(\operatorname{Int}(A))$ denote the closure (the interior) of $A$ in the natural topology $\mathcal{T}$ on the real line.

(C) 2015 Mathematical Institute, Slovak Academy of Sciences.

2010 Mathematics Subject Classification: 28A05, 54A10, 54C50, 54E52.

Keywords: density point, density topology. 
Recall that $x$ is a density point of a measurable set $A$ if and only if

$$
\lim _{h \rightarrow 0^{+}} \frac{m(A \cap[x-h, x+h])}{2 h}=1 .
$$

The set of all density points of a measurable set $A$ will be denoted by $\Phi_{d}(A)$.

Let us observe that the condition given above is equivalent to any of the following:

or

$$
\lim _{n \rightarrow \infty} \frac{m\left(A \cap\left[x-\frac{1}{n}, x+\frac{1}{n}\right]\right)}{\frac{2}{n}}=1
$$

$$
\lim _{n \rightarrow \infty} m(n \cdot(A-x) \cap[-1,1])=2
$$

or

$$
\left\{\chi_{(n \cdot(A-x)) \cap[-1,1]}\right\}_{n \in \mathbb{N}} \text { converges in measure to } \chi_{[-1,1]} \text {. }
$$

Knowing this condition and using the Riesz theorem, the definition of the density point of a measurable set $A$ can be given in terms of convergence almost everywhere of characteristic functions of dilations of the set $A$. Namely, $x$ is a density point of a measurable set $A$ if and only if for each increasing sequence $\left\{n_{m}\right\}_{m \in \mathbb{N}}$ of positive integers there exists a subsequence $\left\{n_{m_{p}}\right\}_{p \in \mathbb{N}}$ such that the sequence of characteristic functions $\left\{\chi_{\left(n_{m_{p}} \cdot(A-x)\right) \cap[-1,1]}\right\}_{p \in \mathbb{N}}$ converges almost everywhere to $\chi_{[-1,1]}$. The new definition allowed for the study of a subtler properties of the notion of the density point and the density topology, their various modifications and most of all category analogues (see [2], [13, [14 and [15). For other generalizations of the density point concept such as $\psi$-density, $f$-density and $\mathcal{A}_{d}$-density see [5], [19] and [24].

In our paper we present the generalization of the approach given in 23 where in the definition of so called c-density point, both measure and category are involved. The ideas from [23] were generalized and developed further in a series of papers [21, 22, and others.

However in the present definition of a density, defining the convergence of dilations of a set, instead of the $\sigma$-ideal of sets of measure zero, we use a smaller $\sigma$-ideal of microscopic sets. For the survey article on the microscopic sets see [1] and [10].

We shall say that some property holds $\mathcal{I}$-almost everywhere (in abbr. $\mathcal{I}$-a.e.) if the set of all points which do not have this property is of the first category.

Let $f, f_{n}, n \in \mathbb{N}$ be real functions defined on $\mathbb{R}$.

Definition 1 ([17]). We say that the sequence $\left\{f_{n}\right\}_{n \in \mathbb{N}}$ converges with respect to the $\sigma$-ideal $\mathcal{I}$ to a function $f$ (in abbr. $f_{n} \underset{n \rightarrow \infty}{\stackrel{\mathcal{I}}{\rightarrow}} f$ ) if and only if every subsequence $\left\{f_{n_{m}}\right\}_{m \in \mathbb{N}}$ of $\left\{f_{n}\right\}_{n \in \mathbb{N}}$ has a subsequence $\left\{f_{n_{m_{p}}}\right\}_{p \in \mathbb{N}}$ converging to $f \mathcal{I}$-a.e. 


\section{DENSITY TOPOLOGY INVOLVING MICROSCOPIC SETS AND CATEGORY}

The category analogue of the density point, given by Wilczynski, is defined as follows:

Definition 2 ([18]). We say that 0 is an $\mathcal{I}$-density point of a set $A$ having the Baire property if and only if

meaning

$$
\chi_{n \cdot A \cap[-1,1]} \underset{n \rightarrow \infty}{\stackrel{\mathcal{I}}{\longrightarrow}} \chi_{[-1,1]},
$$

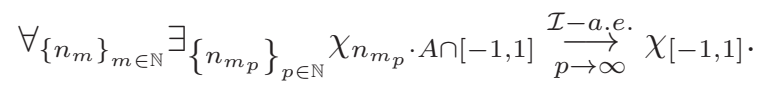

This is equivalent to the fact that for each subsequence $\left\{n_{m}\right\}_{m \in \mathbb{N}}$ of natural numbers there exists a subsequence $\left\{n_{m_{p}}\right\}_{p \in \mathbb{N}}$ such that

$$
[-1,1] \backslash \lim \inf _{p \rightarrow \infty}\left(n_{m_{p}} \cdot A\right) \in \mathcal{I} .
$$

We say that $x$ is an $\mathcal{I}$-density point of $A$ having the Baire property if 0 is an $\mathcal{I}$-density point of $A-x$.

The set of all $\mathcal{I}$-density points of $A$, for $A \in \mathcal{B}$, will be denoted by $\Phi_{\mathcal{I}}(A)$.

It is clear that in the definition mentioned above we can replace the set $A$ with any open set $G$ such that $A=G \triangle P$, where $P \in \mathcal{I}$. Particularly, we can require $G$ to be the regular open set.

In [23], the author introduced the notion of $c$-density in the following way:

Definition 3 ([23]). We say that 0 is a $c$-density point of $A \in \mathcal{B}$ if and only if

$$
\lim _{h \rightarrow \infty} \frac{m(G \cap[-h, h])}{2 h}=1,
$$

where $G$ is a regular open set such that $A \sim G$.

Remark 1. The following conditions are obviously equivalent:

(i) 0 is a $c$-density point of,

(ii) 0 is a density point of $G$,

(iii) $\lim _{n \rightarrow \infty} \frac{m\left(G \cap\left[-\frac{1}{n}, \frac{1}{n}\right]\right)}{\frac{2}{n}}=1$,

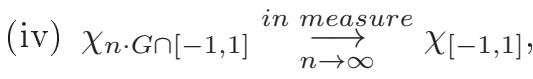

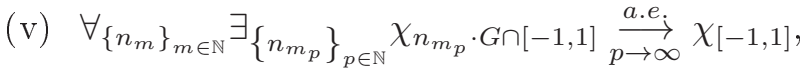

(vi) $\forall_{\left\{n_{m}\right\}_{m \in \mathbb{N}}} \exists_{\left\{n_{m_{p}}\right\}_{p \in \mathbb{N}}} m\left([-1,1] \backslash \liminf \inf _{p \rightarrow \infty}\left(n_{m_{p}} \cdot G\right)\right)=0$.

It is known ([23]) that the notion of $c$-density point leads us to the operator $\Phi_{c}: \mathcal{B} \longrightarrow 2^{\mathbb{R}}$ defined as follows

$$
\Phi_{c}(A)=\{x \in \mathbb{R}: x \text { is a } c \text {-density point of } A\} \text { for } A \in \mathcal{B},
$$




\section{E. WAGNER-BOJAKOWSKA - W. WILCZYŃSKI - W. WOJDOWSKI}

$\Phi_{c}$ is so-called lower density and the family

$$
\mathcal{T}_{c}=\left\{A \in \mathcal{B}: A \subset \Phi_{c}(A)\right\}=\left\{\Phi_{c}(A) \backslash P: A \in \mathcal{B}, P \in \mathcal{I}\right\}
$$

is a topology on the real line, which is incomparable with the classical density topology and with the $\mathcal{I}$-density topology.

In this paper, we introduce another notion of density, replacing the convergence a.e. with the convergence except for a microscopic set, i.e., in (v) the $\sigma$-ideal of sets of Lebesgue measure zero is replaced with the $\sigma$-ideal of microscopic sets.

Definition 4 ([1]). The set $A \subset \mathbb{R}$ is microscopic if for each $\epsilon>0$ there exists a sequence $\left\{I_{n}\right\}_{n \in \mathbb{N}}$ of intervals such that

$$
A \subset \bigcup_{n=1}^{\infty} I_{n} \text { and } m\left(I_{n}\right) \leqslant \epsilon^{n} \quad \text { for } n \in \mathbb{N} .
$$

In [1, it is proved that the family $\mathcal{M}$ of microscopic sets is a $\sigma$-ideal on the real line, which is situated between countable sets and sets of Lebesgue measure zero, and the classical Cantor set is not microscopic.

We shall say that some property holds $\mu$-a.e. if the set of all points, which do not have this property, is microscopic.

Let $G$ be a regular open set.

Definition 5. We say that 0 is a $c_{\mu}$-density point of $G$ if and only if for each increasing sequence $\left\{n_{m}\right\}_{m \in \mathbb{N}}$ of natural numbers there exists a subsequence $\left\{n_{m_{p}}\right\}_{p \in \mathbb{N}}$ such that

$$
\chi_{n_{m_{p}} \cdot G \cap[-1,1]} \underset{p \rightarrow \infty}{\stackrel{\mu-a . e .}{\longrightarrow}} \chi_{[-1,1]},
$$

i.e.,

$$
\forall\left\{n_{m}\right\}_{m \in \mathbb{N}} \exists\left\{n_{m_{p}}\right\}_{p \in \mathbb{N}}[-1,1] \backslash \lim \inf _{p \rightarrow \infty}\left(n_{m_{p}} \cdot G\right) \in \mathcal{M} .
$$

We shall say that zero is a right $c_{\mu}$-density point of a regular open set $G$ if

$$
\forall\left\{n_{m}\right\}_{m \in \mathbb{N}} \exists\left\{n_{m_{p}}\right\}_{p \in \mathbb{N}}[0,1] \backslash \lim \inf _{p \rightarrow \infty}\left(n_{m_{p}} \cdot G\right) \in \mathcal{M} .
$$

In the analogous way, we can define that zero is a left $c_{\mu}$-density point of a regular open set $G$. Obviously, zero is a $c_{\mu}$-density point of $G$ if and only if it is a right and left $c_{\mu}$-density point of $G$.

We shall say that $x$ is a $c_{\mu}$-density point of $G$ if 0 is a $c_{\mu}$-density point of the set $G-x$.

Definition 6. We say that $x$ is a $c_{\mu}$-density point of $A \in \mathcal{B}$ if and only if $x$ is a $c_{\mu}$-density point of $G$, where $G$ is a regular open set such that $A \sim G$. 


\section{DENSITY TOPOLOGY INVOLVING MICROSCOPIC SETS AND CATEGORY}

We shall say that $x$ is a dispersion ( $\mathcal{I}$-dispersion, $c$-dispersion, $c_{\mu}$-dispersion) point of $A$ if $x$ is a density ( $\mathcal{I}$-density, $c$-density, $c_{\mu}$-density) point of $\mathbb{R} \backslash A$.

We shall prove that $c_{\mu}$-density defined as above is really a density, i.e., leads us to the abstract density topology introduced by the operator called lower density.

Put

$$
\Phi_{c_{\mu}}(A)=\left\{x \in \mathbb{R}: x \text { is a } c_{\mu} \text {-density point of } A\right\} \text { for } A \in \mathcal{B} \text {. }
$$

Clearly, $\Phi_{c_{\mu}}(A)=\Phi_{c_{\mu}}(G)$ if $A \in \mathcal{B}$ and $G$ is a regular open set such that $A \sim G$. It is easily seen that the operator $\Phi_{c_{\mu}}$ is monotonic, i.e., if $A, B \in \mathcal{B}$ and $A \subset B$, then $\Phi_{c_{\mu}}(A) \subset \Phi_{c_{\mu}}(B)$.

Theorem 1. For every $A, B \in \mathcal{B}$

1) $\Phi_{c_{\mu}}(A) \sim A$,

2) if $A \sim B$, then $\Phi_{c_{\mu}}(A)=\Phi_{c_{\mu}}(B)$,

3) $\Phi_{c_{\mu}}(\emptyset)=\emptyset, \Phi_{c_{\mu}}(\mathbb{R})=\mathbb{R}$,

4) $\Phi_{c_{\mu}}(A \cap B)=\Phi_{c_{\mu}}(A) \cap \Phi_{c_{\mu}}(B)$.

P r o of. 1) Let $A=G \triangle P$, where $G$ is a regular open set and $P \in \mathcal{I}$. It is easily seen that

$$
G \subset \Phi_{c_{\mu}}(G) \subset \bar{G}
$$

SO

$$
G \triangle \Phi_{c_{\mu}}(G) \subset \bar{G} \backslash G=F r G .
$$

As $\operatorname{Fr} G$ is nowhere dense set, we obtain $G \sim \Phi_{c_{\mu}}(G)$. Obviously, $A \sim G \sim$ $\Phi_{c_{\mu}}(G)=\Phi_{c_{\mu}}(A)$, hence $A \sim \Phi_{c_{\mu}}(A)$.

2) Let $A=G_{1} \triangle P_{1}, B=G_{2} \triangle P_{2}$, where $G_{1}, G_{2}$ are regular open sets and $P_{1}, P_{2} \in \mathcal{I}$. If $A \sim B$, then we have $G_{1}=G_{2}$. Consequently, $B=G_{1} \triangle P_{2}$ and

$$
\Phi_{c_{\mu}}(A)=\Phi_{c_{\mu}}\left(G_{1}\right)=\Phi_{c_{\mu}}(B) .
$$

3) It is a consequence of the definition of $\Phi_{c_{\mu}}$.

4) Obviously, $\Phi_{c_{\mu}}(A \cap B) \subset \Phi_{c_{\mu}}(A) \cap \Phi_{c_{\mu}}(B)$.

Now, let $x_{0} \in \Phi_{c_{\mu}}(A) \cap \Phi_{c_{\mu}}(B)$. Suppose that $A=G_{1} \triangle P_{1}, B=G_{2} \triangle P_{2}$, where $G_{1}, G_{2}$ are regular open sets and $P_{1}, P_{2} \in \mathcal{I}$. As $x_{0} \in \Phi_{c_{\mu}}(A), x_{0}$ is a $c_{\mu}$-density point of $G_{1}$. Let $\left\{n_{m}\right\}_{m \in \mathbb{N}}$ be an arbitrary subsequence of natural numbers. There exists a subsequence $\left\{n_{m_{p}}\right\}_{p \in \mathbb{N}}$ such that

$$
\chi_{n_{m_{p}} \cdot\left(G_{1}-x_{0}\right) \cap[-1,1]}(x) \underset{p \rightarrow \infty}{\longrightarrow} \chi_{[-1,1]}(x)
$$

for all $x$ except for some microscopic set $Q_{1}$. As $x_{0} \in \Phi_{c_{\mu}}\left(G_{2}\right)$, there exists a subsequence $\left\{n_{m_{p_{k}}}\right\}_{k \in \mathbb{N}}$ of the sequence $\left\{n_{m_{p}}\right\}_{p \in \mathbb{N}}$ such that

$$
\chi_{n_{m_{p_{k}}} \cdot\left(G_{2}-x_{0}\right) \cap[-1,1]}(x) \underset{k \rightarrow \infty}{\longrightarrow} \chi_{[-1,1]}(x)
$$

for all $x$ except for some microscopic set $Q_{2}$. 
Obviously,

$$
\chi_{n_{m_{p_{k}}} \cdot\left(\left(G_{1} \cap G_{2}\right)-x_{0}\right) \cap[-1,1]}(x) \underset{k \rightarrow \infty}{\longrightarrow} \chi_{[-1,1]}(x)
$$

for all $x \in \mathbb{R} \backslash\left(Q_{1} \cup Q_{2}\right)$ and $Q_{1} \cup Q_{2}$ is a microscopic set. From the arbitrariness of the sequence $\left\{n_{m}\right\}_{m \in \mathbb{N}}$ we have $x_{0} \in \Phi_{c_{\mu}}\left(G_{1} \cap G_{2}\right)$. Obviously, $G_{1} \cap G_{2}$ is a regular open set and $(A \cap B) \triangle\left(G_{1} \cap G_{2}\right) \in \mathcal{I}$, so $x_{0} \in \Phi_{c_{\mu}}(A \cap B)$.

From the last theorem it follows that the operator $\Phi_{c_{\mu}}: \mathcal{B} \longrightarrow \mathcal{B}$ is a lower density ([19]). Obviously, the first property is analogous to the Lebesgue Density Theorem, which holds for metric density.

Observe that if $B$ is an arbitrary residual set, even microscopic (see [10, Lemma 2.2]), then $B \sim \mathbb{R}$, so $\Phi_{c_{\mu}}(B)=\Phi_{c_{\mu}}(\mathbb{R})=\mathbb{R}$.

The sets $\bigcup_{n=1}^{\infty}\left[a_{n}, b_{n}\right]$ or $\bigcup_{n=1}^{\infty}\left(a_{n}, b_{n}\right)$ are called right interval sets at zero if $b_{n+1}<a_{n}<b_{n}$ for $n \in \mathbb{N}$ and $\lim _{n \rightarrow \infty} a_{n}=0$. We shall now prove that there exists a right interval set $G$ such that zero is a right $c_{\mu}$-density point of $G$.

LEMMA 1. If $G=\bigcup_{n=1}^{\infty}\left(d_{n+1}, c_{n}\right)$ is a right interval set such that

(i) $\frac{d_{n}-c_{n}}{d_{n}} \underset{n \rightarrow \infty}{\searrow} 0$,

(ii) $\frac{d_{n+1}}{d_{n}} \underset{n \rightarrow \infty}{\searrow} 0$,

then zero is a right $c_{\mu}$-density point of $G$.

P r o o f. It follows easily from the proof in [2, Lemma 2.1.6], since countable sets are microscopic.

As it was mentioned earlier, each microscopic set has Lebesgue measure zero, so for arbitrary set $A \in \mathcal{B}$ and $x \in \mathbb{R}$, if $x$ is a $c_{\mu}$-density point of $A$ then $x$ is also a $c$-density point of $A$, i.e.,

$$
\Phi_{c_{\mu}}(A) \subset \Phi_{c}(A)
$$

for $A \in \mathcal{B}$. Now, we shall prove that there exists a set $A \in \mathcal{B}$ for which this inclusion is proper. For this purpose, we need an auxiliary lemma.

Let $A^{\prime}=\mathbb{R} \backslash A$ for $A \subset \mathbb{R}$.

LEMMA 2. Let $\eta \in(0,1)$ and let $\left\{c_{n}\right\}_{n \in \mathbb{N}}$ be a decreasing sequence tending to zero such that $\frac{c_{n+1}}{c_{n}}>\eta$ for $n \in \mathbb{N}$. If $\left\{A_{n}\right\}_{n \in \mathbb{N}}$ is a sequence of sets fulfilling conditions

(i) $A_{n} \subset\left[c_{n+1}, c_{n}\right]$ for $n \in \mathbb{N}$,

(ii) $\frac{m\left(A_{n}\right)}{c_{n}-c_{+1}} \underset{n \rightarrow \infty}{\nearrow} 1$,

then the right-hand density at zero of the set $A=\bigcup_{n=1}^{\infty} A_{n}$ is equal to 1 . 
Proof. Put

$$
\alpha_{n}=\frac{m\left(\left[c_{n+1}, c_{n}\right] \backslash A_{n}\right)}{c_{n}-c_{n+1}} \text { for } n \in \mathbb{N} .
$$

From (ii) we obtain $\alpha_{n} \underset{n \rightarrow \infty}{\searrow} 0$.

Let $h \in\left(0, c_{1}\right]$. Then, there exists $n_{0} \in \mathbb{N}$ such that

SO

$$
c_{n_{0}+1}<h \leqslant c_{n_{0}},
$$

$$
\begin{aligned}
& \frac{m\left(A^{\prime} \cap[0, h]\right)}{h} \leqslant \frac{m\left(A^{\prime} \cap\left[0, c_{n_{0}}\right]\right)}{c_{n_{0}+1}}=\frac{\sum_{k=n_{0}}^{\infty} m\left(\left[c_{k+1}, c_{k}\right] \backslash A_{k}\right)}{c_{n_{0}+1}}= \\
& \quad \frac{\sum_{k=n_{0}}^{\infty} \alpha_{k}\left(c_{k}-c_{k+1}\right)}{c_{n_{0}+1}} \leqslant \frac{\alpha_{n_{0}} \sum_{k=n_{0}}^{\infty}\left(c_{k}-c_{k+1}\right)}{c_{n_{0}+1}}=\frac{\alpha_{n_{0}} \cdot c_{n_{0}}}{c_{n_{0}+1}}<\alpha_{n_{0}} \cdot \frac{1}{\eta} .
\end{aligned}
$$

If $h$ tends to zero, then $n_{0}$ tends to infinity and so $\alpha_{n_{0}}$ tends to zero. Consequently, the right-hand density at zero of the set $A^{\prime}$ is equal to zero, hence zero is the right-hand density point of $A$.

TheOREM 2. There exists a regular open set $G$ such that zero is a density, c-density and $\mathcal{I}$-density point of $G$ but not a $c_{\mu}$-density point of $G$, i.e.,

$$
0 \in \Phi_{d}(G) \cap \Phi_{c}(G) \cap \Phi_{\mathcal{I}}(G) \backslash \Phi_{c_{\mu}}(G) .
$$

P r o o f. Let $C$ denote the classical Cantor set. Let $G_{1}$ be a component interval of $[0,1] \backslash C$, concentric with $[0,1]$, which is removed in the first step of the construction of $C$. Let $G_{2}$ denote the union of the component intervals of $[0,1] \backslash C$, which are removed in the first and second steps. And so on. Let $G_{n}$ be the union of the component intervals of $[0,1] \backslash C$, which are removed in the steps $1, \ldots, n$, for $n \in \mathbb{N}$.

Put

$$
G=(-\infty, 0) \cup \bigcup_{n=1}^{\infty}\left(\frac{1}{2^{n}} G_{n}+\frac{1}{2^{n}}\right) .
$$

Obviously, $G$ is a regular open set.

As $m\left(G_{n}\right) \underset{n \rightarrow \infty}{\longrightarrow} 1$, we have

$$
\frac{m\left(\frac{1}{2^{n}} G_{n}+\frac{1}{2^{n}}\right)}{\frac{1}{2^{n}}} \underset{n \rightarrow \infty}{\longrightarrow} 1 \text { and } \frac{1}{2^{n}} G_{n}+\frac{1}{2^{n}} \subset\left[\frac{1}{2^{n}}, \frac{1}{2^{n-1}}\right] \quad \text { for } n \in \mathbb{N},
$$

so from Lemma 2, (used for $c_{n}=\frac{1}{2^{n-1}}$ and $A_{n}=\frac{1}{2^{n}} G_{n}+\frac{1}{2^{n}}$ ), zero is a density point of $G$ and consequently, $0 \in \Phi_{d}(G) \cap \Phi_{c}(G)$.

Now, we shall prove that zero is an $\mathcal{I}$-density point of $G$. According to [2, Theorem 2.2.2, condition (ii)], it is sufficient to prove that for each increasing sequence $\left\{t_{k}\right\}_{k \in \mathbb{N}}$ of positive numbers tending to infinity there exists a subsequence $\left\{t_{k_{p}}\right\}_{p \in \mathbb{N}}$ such that

$$
\lim \inf _{p \rightarrow \infty}\left(t_{k_{p}} \cdot G\right) \cap[-1,1]=\bigcup_{m=1}^{\infty} \bigcap_{p=m}^{\infty}\left(t_{k_{p}} \cdot G\right) \cap[-1,1]
$$




\section{E. WAGNER-BOJAKOWSKA - W. WILCZYŃSKI - W. WOJDOWSKI}

is a residual set in $[-1,1]$.

Let $\left\{t_{k}\right\}_{k \in \mathbb{N}}$ be an increasing sequence of positive numbers tending to infinity, $t_{1}>1$. Put

$$
h_{k}=\frac{1}{t_{k}}
$$

for $k \in \mathbb{N}$. Then, for each $k \in \mathbb{N}$ there exists exactly one $n_{k} \in \mathbb{N}$ such that

$$
h_{k} \in\left[\frac{1}{2^{n_{k}+1}}, \frac{1}{2^{n_{k}}}\right] .
$$

Hence,

$$
1 \leqslant h_{k} \cdot 2^{n_{k}+1} \leqslant 2,
$$

so the sequence $\left\{h_{k} \cdot 2^{n_{k}+1}\right\}_{k \in \mathbb{N}}$ is bounded and contains a subsequence $\left\{h_{k_{p}} \cdot 2^{n_{k_{p}}+1}\right\}_{p \in \mathbb{N}}$ convergent to some number $g \in[1,2]$.

Clearly,

so

$$
h_{k_{p}} \cdot 2^{n_{k_{p}}} \underset{p \rightarrow \infty}{\longrightarrow} \frac{g}{2}
$$

and $\frac{2}{g} \in[1,2]$.

$$
\frac{1}{h_{k_{p}} \cdot 2^{n_{k_{p}}}} \underset{p \rightarrow \infty}{\longrightarrow} \frac{2}{g}
$$

We shall prove that $\lim \inf \left(t_{k_{p}} \cdot G\right) \cap(0,1)$ is a set residual in $(0,1)$.

Put

$$
G_{0}=\bigcup_{n=1}^{\infty} G_{n}
$$

and

$$
B=\bigcup_{n=1}^{\infty}\left(\frac{1}{2^{n}} G_{0}+\frac{1}{2^{n}}\right)
$$

Clearly, $G_{0}$ is open and dense in $[0,1]$, so $B$ is also.

Observe that

$$
\begin{aligned}
\lim \inf _{p \rightarrow \infty}\left(t_{k_{p}} \cdot G\right) \cap(0,1) & =\bigcup_{m=1}^{\infty} \bigcap_{p=m}^{\infty}\left(t_{k_{p}} \cdot G\right) \cap(0,1) \\
& =\bigcup_{m=1}^{\infty} \bigcap_{p=m}^{\infty}\left(\frac{1}{h_{k_{p}}} \cdot G\right) \cap(0,1) \supset\left(\frac{2}{g} \cdot B\right) \cap(0,1) .
\end{aligned}
$$

Let $x \in\left(\frac{2}{g} \cdot B\right) \cap(0,1)$. Since $B$ is open, $x$ belongs to some component interval of $\left(\frac{2}{g} \cdot B\right) \cap(0,1)$. Hence, there exist $n_{0}, m_{0} \in \mathbb{N}$ such that

$$
x \in \frac{2}{g}\left(\frac{1}{2^{n_{0}}} G_{m_{0}}+\frac{1}{2^{n_{0}}}\right) .
$$


Let $n_{k_{p}} \geqslant m_{0}$. Then,

$$
\begin{aligned}
\frac{1}{h_{k_{p}}} \cdot G \cap(0,1) & =\frac{2^{n_{k_{p}}}}{h_{k_{p}} \cdot 2^{n_{k_{p}}}} \cdot G \cap(0,1) \\
& =\frac{1}{h_{k_{p}} \cdot 2^{n_{k_{p}}}} \cdot \bigcup_{m=1}^{\infty}\left(\frac{2^{n_{k_{p}}}}{2^{m}} G_{m}+\frac{2^{n_{k_{p}}}}{2^{m}}\right) \cap(0,1) \\
& =\frac{1}{h_{k_{p}} \cdot 2^{n_{k_{p}}}} \cdot \bigcup_{m=n_{k_{p}}+1}^{\infty}\left(\frac{2^{n_{k_{p}}}}{2^{m}} G_{m}+\frac{2^{n_{k_{p}}}}{2^{m}}\right) \\
& =\frac{1}{h_{k_{p}} \cdot 2^{n_{k_{p}}}} \cdot \bigcup_{m=n_{k_{p}}+1}^{\infty}\left(\frac{1}{2^{m-n_{k_{p}}}} G_{m}+\frac{1}{2^{m-n_{k_{p}}}}\right) \\
& \supset \frac{1}{h_{k_{p}} \cdot 2^{n_{k_{p}}}} \cdot\left(\frac{1}{2^{n_{0}}} G_{m_{0}}+\frac{1}{2^{n_{0}}}\right) \underset{p \rightarrow \infty}{\longrightarrow} \cdot \frac{2}{g} \cdot\left(\frac{1}{2^{n_{0}}} G_{m_{0}}+\frac{1}{2^{n_{0}}}\right)
\end{aligned}
$$

according to (3).

Hence, using (2) and (5), we obtain $x \in\left(t_{k_{p}} \cdot G\right) \cap(0,1)$ for each $p$ sufficiently large and such that $n_{k_{p}} \geqslant m_{0}$, so (4) is fulfilled.

Since $B$ is open and dense in $[0,1]$ and $\frac{2}{g} \geqslant 1$, from (4) it follows that the set $\liminf _{p \rightarrow \infty}\left(t_{k_{p}} \cdot G\right) \cap(0,1)$ is residual in $[0,1]$. Consequently, using (1) we obtain that zero is an $\mathcal{I}$-density point of $G$.

Now, we shall prove that zero is not a $c_{\mu}$-density point of $G$. Put $n_{m}=2^{m}$ for $m \in \mathbb{N}$.

Let us observe that $[0,1] \backslash \liminf _{m \rightarrow \infty}\left(2^{m} \cdot G\right)=\limsup _{m \rightarrow \infty}\left([0,1] \backslash 2^{m} \cdot G\right)$ is not a microscopic set. Indeed, we have

$$
\begin{aligned}
\lim \sup _{m \rightarrow \infty}\left([0,1] \backslash 2^{m} \cdot G\right) & =\bigcap_{k=1}^{\infty} \bigcup_{m=k}^{\infty}\left([0,1] \backslash 2^{m} \cdot \bigcup_{n=1}^{\infty}\left(\frac{1}{2^{n}} G_{n}+\frac{1}{2^{n}}\right)\right) \\
& =\bigcap_{k=1}^{\infty} \bigcup_{m=k}^{\infty}\left([0,1] \backslash \bigcup_{n=1}^{\infty}\left(\frac{2^{m}}{2^{n}} G_{n}+\frac{2^{m}}{2^{n}}\right)\right) \\
& =\bigcap_{k=1}^{\infty} \bigcup_{m=k}^{\infty}\left([0,1] \backslash \bigcup_{n=m+1}^{\infty}\left(\frac{1}{2^{n-m}} G_{n}+\frac{1}{2^{n-m}}\right)\right) \\
& \supset \bigcap_{k=1}^{\infty} \bigcup_{m=k}^{\infty}\left([0,1] \backslash \bigcup_{n=m+1}^{\infty}\left(\frac{1}{2^{n-m}} G_{0}+\frac{1}{2^{n-m}}\right)\right)
\end{aligned}
$$

because $G_{n} \subset G_{0}$ for each $n \in \mathbb{N}$. 
Simultaneously,

$$
\bigcup_{n=m+1}^{\infty}\left(\frac{1}{2^{n-m}} G_{0}+\frac{1}{2^{n-m}}\right)=\bigcup_{n=1}^{\infty}\left(\frac{1}{2^{n}} G_{0}+\frac{1}{2^{n}}\right)=B
$$

and

so

$$
[0,1] \backslash B=\bigcup_{n=1}^{\infty}\left(\frac{1}{2^{n}} C+\frac{1}{2^{n}}\right)
$$

$$
\bigcup_{n=1}^{\infty}\left(\frac{1}{2^{n}} C+\frac{1}{2^{n}}\right) \subset \lim \sup _{m \rightarrow \infty}\left([0,1] \backslash 2^{m} \cdot G\right) .
$$

Consequently, $[0,1] \backslash \liminf _{m \rightarrow \infty}\left(2^{m} \cdot G\right)$ is not a microscopic set, as the Cantor set and also all sets of the form $\frac{1}{2^{n}} C+\frac{1}{2^{n}}, n \in \mathbb{N}$, are not microscopic. An analogous result is obtained for arbitrary subsequence of the sequence $\left\{2^{m}\right\}_{m \in \mathbb{N}}$. Hence, zero is not a $c_{\mu}$-density point of $G$, i.e., $0 \notin \Phi_{c_{\mu}}(G)$.

THEOREM 3. There exists a right interval set $A$ such that zero is a $c_{\mu}$-dispersion point but not an $\mathcal{I}$-dispersion point of $A$.

P r o of. Let $n_{1} \in \mathbb{N}$ (we can assume $n_{1}=1$ ). Put

$$
I_{1}^{1}=\left(\frac{1}{2 n_{1}}, \frac{1}{n_{1}}\right) .
$$

Obviously, $m\left(I_{1}^{1}\right)=\frac{1}{2 n_{1}} \cdot\left(\frac{1}{1}\right)^{1 !}$.

Let $n_{2}$ be a natural number such that

$$
\frac{1}{n_{2}}<\frac{1}{2 n_{1}} \cdot\left(\frac{1}{2}\right)^{2 !}
$$

In the interval $\left(\frac{1}{2 n_{2}}, \frac{1}{n_{2}}\right)$, we construct two open intervals $I_{2}^{1}, I_{2}^{2}$ with right end-points equal to

and with lengths equal to

$$
\frac{1}{n_{2}}, \quad \frac{1}{2 n_{2}}\left(1+\frac{1}{2}\right)
$$

$$
\frac{1}{2 n_{2}}\left(\frac{1}{3}\right)^{3 !}, \quad \frac{1}{2 n_{2}}\left(\frac{1}{4}\right)^{4 !}
$$

respectively.

Now, let $n_{3}$ be a natural number such that

$$
\frac{1}{n_{3}}<\frac{1}{2 n_{2}} \cdot\left(\frac{1}{5}\right)^{5 !}
$$

In the interval $\left(\frac{1}{2 n_{3}}, \frac{1}{n_{3}}\right)$, we construct three open intervals $I_{3}^{1}, I_{3}^{2}, I_{3}^{3}$ with 
right end-points equal to

and with lengths equal to

$$
\frac{1}{n_{3}}=\frac{1}{2 n_{3}} \cdot 2, \quad \frac{1}{2 n_{3}}\left(1+\frac{2}{3}\right), \quad \frac{1}{2 n_{3}}\left(1+\frac{1}{3}\right)
$$

$$
\frac{1}{2 n_{3}}\left(\frac{1}{6}\right)^{6 !}, \quad \frac{1}{2 n_{3}}\left(\frac{1}{7}\right)^{7 !}, \quad \frac{1}{2 n_{3}}\left(\frac{1}{8}\right)^{8 !}
$$

respectively.

Now, we proceed by induction. Suppose that we have defined natural numbers $n_{1}, n_{2}, \ldots, n_{k}$, and $\frac{k(k+1)}{2}$ open intervals

$$
I_{1}^{1}, I_{2}^{1}, I_{2}^{2}, I_{3}^{1}, I_{3}^{2}, I_{3}^{3}, \ldots, I_{k}^{1}, I_{k}^{2}, \ldots, I_{k}^{k} .
$$

The lengths of the intervals in $k$ th group $\left(I_{k}^{1}, I_{k}^{2}, \ldots, I_{k}^{k}\right)$ are equal to

$\frac{1}{2 n_{k}} \cdot\left(\frac{1}{\frac{(k-1)(k+2)}{2}+1}\right)^{\left.\frac{(k-1)(k+2)}{2}+1\right) !}$,

$\frac{1}{2 n_{k}} \cdot\left(\frac{1}{\frac{(k-1)(k+2)}{2}+2}\right)^{\left.\frac{(k-1)(k+2)}{2}+2\right) !}, \ldots, \frac{1}{2 n_{k}} \cdot\left(\frac{1}{\frac{(k-1)(k+2)}{2}+k}\right)^{\left(\frac{(k-1)(k+2)}{2}+k\right) !}$,

and the right end-points are

$$
\frac{1}{n_{k}}=\frac{1}{2 n_{k}} \cdot 2, \frac{1}{2 n_{k}}\left(1+\frac{k-1}{k}\right), \ldots, \frac{1}{2 n_{k}}\left(1+\frac{2}{k}\right), \frac{1}{2 n_{k}}\left(1+\frac{1}{k}\right),
$$

respectively.

Let $n_{k+1}$ be a natural number such that

$$
\frac{1}{n_{k+1}}<\frac{1}{2 n_{k}}\left(\frac{1}{\frac{k(k+3)}{2}}\right)^{\left(\frac{k(k+3)}{2}\right) !} .
$$

In the interval $\left(\frac{1}{2 n_{k+1}}, \frac{1}{n_{k+1}}\right)$, we construct the open intervals

$$
I_{k+1}^{1}, I_{k+1}^{2}, \ldots, I_{k+1}^{k+1}
$$

with lengths equal to

$$
\frac{1}{2 n_{k+1}} \cdot\left(\frac{1}{\frac{k(k+3)}{2}+1}\right)^{\left(\frac{k(k+3)}{2}+1\right) !}, \ldots, \frac{1}{2 n_{k+1}} \cdot\left(\frac{1}{\frac{k(k+3)}{2}+k+1}\right)^{\left(\frac{k(k+3)}{2}+k+1\right) !}
$$

and with right end-points

$$
\frac{1}{n_{k+1}}, \frac{1}{2 n_{k+1}}\left(1+\frac{k}{k+1}\right), \ldots, \frac{1}{2 n_{k+1}}\left(1+\frac{2}{k+1}\right), \frac{1}{2 n_{k+1}}\left(1+\frac{1}{k+1}\right),
$$

respectively. 
Put

$$
A=\bigcup_{k=1}^{\infty} \bigcup_{j=1}^{k} I_{k}^{j}
$$

Clearly, $A$ is a right interval set. We shall first prove that zero is a $c_{\mu}$-dispersion point of $A$. For this purpose, we shall prove that for each increasing sequence $\left\{i_{m}\right\}_{m \in \mathbb{N}}$ of natural numbers there exists a subsequence $\left\{i_{m_{p}}\right\}_{p \in \mathbb{N}}$ such that

$$
\lim \sup _{p \rightarrow \infty}\left(i_{m_{p}} \cdot A\right) \cap[0,1]=[0,1] \cap \bigcap_{r=1}^{\infty} \bigcup_{p=r}^{\infty} i_{m_{p}} \cdot A \in \mathcal{M} .
$$

Let $\left\{i_{m}\right\}_{m \in \mathbb{N}}$ be an arbitrary increasing sequence of natural numbers. For each $m \in \mathbb{N}$ there exists exactly one $k \in \mathbb{N}$ such that

$$
\frac{1}{2 n_{k}} \leqslant \frac{1}{i_{m}}<\frac{1}{2 n_{k-1}}
$$

(for $k=1$ we put $\frac{1}{2 n_{k-1}}=2$ ).

If $\left\{m \in \mathbb{N}: \frac{1}{2 n_{k}} \leqslant \frac{1}{i_{m}}<\frac{1}{2 n_{k-1}}\right\} \neq \emptyset$, then we choose exactly one element from this set. In such a way, we construct a sequence $\left\{m_{p}\right\}_{p \in \mathbb{N}}$ having the property that for each $k \in \mathbb{N}$, if

$$
\left\{m \in \mathbb{N}: \frac{1}{2 n_{k}} \leqslant \frac{1}{i_{m}}<\frac{1}{2 n_{k-1}}\right\} \neq \emptyset,
$$

then there exists exactly one number $m_{p}$ such that

$$
\frac{1}{2 n_{k}} \leqslant \frac{1}{i_{m_{p}}}<\frac{1}{2 n_{k-1}} .
$$

Observe that the set $\left(i_{m_{p}} \cdot A\right) \cap[0,1]$ consists of at most $k$ intervals $i_{m_{p}} \cdot I_{k}^{1}, \ldots$ $\ldots, i_{m_{p}} \cdot I_{k}^{k}$ and of the second part contained in the interval $i_{m_{p}} \cdot\left[0, \frac{1}{n_{k+1}}\right]$, where $k$ fulfils (8). So,

$$
\left(i_{m_{p}} \cdot A\right) \cap[0,1] \subset\left[0, \frac{i_{m_{p}}}{n_{k+1}}\right] \cup \bigcup_{j=1}^{k} i_{m_{p}} \cdot I_{k}^{j} .
$$

Obviously, from (8) and (6)

$$
m\left(i_{m_{p}} \cdot I_{k}^{j}\right) \leqslant m\left(2 n_{k} \cdot I_{k}^{j}\right)=\left(\frac{1}{\frac{(k-1)(k+2)}{2}+j}\right)^{\left(\frac{(k-1)(k+2)}{2}+j\right) !} .
$$

Using (8) and (7), we obtain

$$
m\left(i_{m_{p}} \cdot\left[0, \frac{1}{n_{k+1}}\right]\right) \leqslant 2 n_{k} \cdot \frac{1}{n_{k+1}} \leqslant\left(\frac{1}{\frac{k(k+3)}{2}}\right)^{\left(\frac{k(k+3)}{2}\right) !},
$$

hence

$$
\bigcup_{p=r}^{\infty}\left(i_{m_{p}} \cdot A\right) \cap[0,1] \subset \bigcup_{p=r}^{\infty}\left(\left[0, \frac{i_{m_{p}}}{n_{k+1}}\right] \cup \bigcup_{j=1}^{k} i_{m_{p}} \cdot I_{k}^{j}\right) \cap[0,1] .
$$


From (8), it follows that for each number $p \in \mathbb{N}$ there exists exactly one number $k(p)$ such that

$$
\frac{1}{2 n_{k(p)}} \leqslant \frac{1}{i_{m_{p}}}<\frac{1}{2 n_{k(p)-1}}
$$

and if $p \rightarrow \infty$, then $k(p) \rightarrow \infty$.

Let $\left\{I_{n}\right\}_{n \in \mathbb{N}}$ be the sequence of intervals

$i_{m_{r}} \cdot I_{k(r)}^{1}, i_{m_{r}} \cdot I_{k(r)}^{2}, \ldots i_{m_{r}} \cdot I_{k(r)}^{k(r)},\left[0, \frac{i_{m_{r}}}{n_{k(r)+1}}\right], i_{m_{r+1}} \cdot I_{k(r+1)}^{1}, i_{m_{r+1}} \cdot I_{k(r+1)}^{2}, \ldots$

Let $\epsilon>0$. Then, there exists $r \in \mathbb{N}$ such that

$$
\frac{1}{\frac{(r-1)(r+2)}{2}+1}<\epsilon
$$

Put $s_{0}=\frac{(r-1)(r+2)}{2}+1$. From $(9)$ the set $\bigcup_{p=r}^{\infty}\left(i_{m_{p}} \cdot A\right) \cap[0,1]$ is covered by the union of intervals $\left\{I_{n}\right\}_{n \in \mathbb{N}}$, and lengths of these intervals are not greater than the elements of the sequence

$$
\left(\frac{1}{s_{0}}\right)^{s_{0} !},\left(\frac{1}{s_{0}+1}\right)^{\left(s_{0}+1\right) !}, \ldots
$$

so, also not greater than the elements of the sequence

$$
\left(\frac{1}{s_{0}}\right)^{s_{0} !},\left(\frac{1}{s_{0}}\right)^{\left(s_{0}+1\right) !}, \ldots
$$

and, finally, than the elements of the sequence

$$
\left(\frac{1}{s_{0}}\right)^{1},\left(\frac{1}{s_{0}}\right)^{2}, \ldots
$$

Consequently, the set $\bigcup_{p=r}^{\infty}\left(i_{m_{p}} \cdot A\right) \cap[0,1]$ is covered by the intervals $\left\{I_{n}\right\}_{n \in \mathbb{N}}$, such that $m\left(I_{n}\right)<\epsilon^{n}$, according to (10). Hence,

is a microscopic set.

$$
\bigcap_{r=1}^{\infty} \bigcup_{p=r}^{\infty} i_{m_{p}} \cdot A \cap[0,1]
$$

Simultaneously, for an arbitrary subsequence $\left\{n_{k_{l}}\right\}_{l \in \mathbb{N}}$ of the sequence $\left\{n_{k}\right\}_{k \in \mathbb{N}}$, the set $\bigcup_{l=r}^{\infty}\left(n_{k_{l}} \cdot A\right)$ is open and dense in the interval $\left[\frac{1}{2}, 1\right]$, so the set

$$
\bigcap_{r=1}^{\infty} \bigcup_{l=r}^{\infty}\left(n_{k_{l}} \cdot A\right)=\lim \sup _{l \rightarrow \infty}\left(n_{k_{l}} \cdot A\right)
$$

is residual on $\left[\frac{1}{2}, 1\right]$. Consequently, zero is not an $\mathcal{I}$-dispersion point of $A$. 
COROLlARY 1. There exists a regular open set $G$ such that zero is a $c_{\mu}$-density point but not an $\mathcal{I}$-density point of $G$, i.e.,

$$
0 \in \Phi_{c_{\mu}}(G) \backslash \Phi_{\mathcal{I}}(G)
$$

Proof. Put

$$
G=\operatorname{Int}(\mathbb{R} \backslash A)
$$

where $A$ is a set from the previous theorem. Then, zero is a $c_{\mu}$-density point of $G$ but not an $\mathcal{I}$-density point of $G$.

Put

$$
\mathcal{T}_{c_{\mu}}=\left\{A \in \mathcal{B}: A \subset \Phi_{c_{\mu}}(A)\right\} .
$$

TheOREM 4. The family $\mathcal{T}_{c_{\mu}}$ is a topology on the real line such that

$$
\mathcal{T} \varsubsetneqq \mathcal{T}_{c_{\mu}} \varsubsetneqq \mathcal{T}_{c} \text {. }
$$

Proof. The only difficulty is to prove that the family $\mathcal{T}_{c_{\mu}}$ is closed under arbitrary unions. For this purpose, it is sufficient to prove that if $\left\{A_{t}\right\}_{t \in T}$ is an arbitrary subfamily of $\mathcal{T}_{c_{\mu}}$, then $\bigcup_{t \in T} A_{t} \in \mathcal{B}$.

For each $t \in T$, there exists a regular open set $G_{t}$ such that $A_{t} \sim G_{t}$. Obviously, there exists a sequence $\left\{t_{n}\right\}_{n \in \mathbb{N}}$ of elements of $T$ such that

Then,

$$
\bigcup_{t \in T} G_{t}=\bigcup_{n=1}^{\infty} G_{t_{n}}
$$

$$
\bigcup_{n=1}^{\infty} G_{t_{n}} \sim \bigcup_{n=1}^{\infty} A_{t_{n}} \subset \bigcup_{t \in T} A_{t} \subset \bigcup_{t \in T} \Phi_{c_{\mu}}\left(A_{t}\right) \subset \bigcup_{t \in T} \bar{G}_{t} \subset \overline{\bigcup_{t \in T} G_{t}}=\overline{\bigcup_{n=1}^{\infty} G_{t_{n}}}
$$

and

$$
\bigcup_{n=1}^{\infty} G_{t_{n}} \sim \overline{\bigcup_{n=1}^{\infty} G_{t_{n}}}, \quad \text { so } \bigcup_{t \in T} A_{t} \in \mathcal{B}
$$

Let $\mathbb{Q}$ denote the set of rational numbers. Obviously, $\mathbb{R} \backslash \mathbb{Q} \in \mathcal{T}_{c_{\mu}} \backslash \mathcal{T}$. Now, let $G_{1}=G \cup\{0\}$, where $G$ is a regular open set from Theorem 2 . Then $G_{1} \in \mathcal{T}_{c} \backslash \mathcal{T}_{c_{\mu}}$.

Remark 2. Analogously as for $\mathcal{I}$-density topology (see [13 Remark 2]), it is clear that $\mathcal{T}_{c_{\mu}}=\left\{\Phi_{c_{\mu}}(A) \backslash P: A \in \mathcal{B}, P \in \mathcal{I}\right\}$.

THEOREM 5. The topology $\mathcal{T}_{c_{\mu}}$ is incomparable with both $\mathcal{T}_{\mathcal{I}}$ and $\mathcal{T}_{d}$ topologies.

P r o of. Let $G$ be a regular open set from Theorem 2. Put

$$
G_{1}=G \cup\{0\} .
$$

Then, $G_{1} \in \mathcal{T}_{\mathcal{I}} \cap \mathcal{T}_{d} \backslash \mathcal{T}_{c_{\mu}}$.

If $G$ is a set from Corollary 1 , then $G \in \mathcal{T}_{c_{\mu}} \backslash \mathcal{T}_{\mathcal{I}}$. 
Let $B$ be a residual set of measure zero. Then, $B \sim \mathbb{R}$, so $\Phi_{c_{\mu}}(B)=\mathbb{R}$ and, consequently, $B \in \mathcal{T}_{c_{\mu}}$. Simultaneously, $B \notin \mathcal{T}_{d}$, as $\Phi_{d}(B)=\emptyset$.

TheOREM 6. If $A \in \mathcal{B}$ and $G$ is regular open set such that $A \sim G$, then

1) $\operatorname{Int}\left(\Phi_{c_{\mu}}(A)\right)=\emptyset$ if and only if $A \in \mathcal{I}$,

2) $\Phi_{c_{\mu}}(A) \backslash G$ is a nowhere dense set of Lebesgue measure zero,

3) $G=\operatorname{Int}\left(\Phi_{c_{\mu}}(A)\right)=\operatorname{Int}(\bar{G})$.

The proof is analogous to the proof of [23, Theorem 3].

Remark 3. Since for each $A \in \mathcal{B}$ we have $\Phi_{c_{\mu}}(A) \sim A$, it is easy to see that, analogously as for the density topology, for arbitrary set $B \subset \mathbb{R}$

$$
\operatorname{Int}_{\mathcal{T}_{c_{\mu}}}(B)=B \cap \Phi_{c_{\mu}}(C),
$$

where $C$ is a measurable (it means having the Baire property) kernel of $B$.

In particular, if $A \in \mathcal{B}$, we have $\operatorname{Int}_{\mathcal{T}_{c_{\mu}}}(A)=A \cap \Phi_{c_{\mu}}(A)$.

Also, a set $A \in \mathcal{B}$ is $\mathcal{T}_{c_{\mu}}$-regular open if and only if $A=\Phi_{c_{\mu}}(D)$ for some $D \in \mathcal{B}$.

THEOREM 7. For arbitrary set $A \subset \mathbb{R}$, the following conditions are equivalent:

1) $A \in \mathcal{I}$,

2) $A$ is $\mathcal{T}_{c_{\mu}}$-nowhere dense set,

3) $A$ is $\mathcal{T}_{c_{\mu}}$-first category set,

4) $A$ is $\mathcal{T}_{c_{\mu}}$-closed and $\mathcal{T}_{c_{\mu}}$-discrete set.

The proof is analogous to the proof in [19, Theorem 2.8], (see also [9]).

\section{TheOREM 8.}

1) The family of $\mathcal{T}_{c_{\mu}}$-Borel sets coincides with the family of sets having the Baire property.

2) Every $\mathcal{T}_{c_{\mu}}$-Borel set is a union of $\mathcal{T}_{c_{\mu}}$-open set and $\mathcal{T}_{c_{\mu}}$-closed set.

3) Every $\mathcal{T}_{c_{\mu}}$-Borel set is $\mathcal{T}_{c_{\mu}}-F_{\sigma \delta}$ set. Proof.

1) The proof is analogous to the proof of Theorem 2.9 in [19].

2) This is clear since for each $A \in \mathcal{B}$ we have $A=\left(A \cap \Phi_{c_{\mu}}(A)\right) \cup\left(A \backslash \Phi_{c_{\mu}}(A)\right)$.

3) If $A \in \mathcal{B}$, then $A=E \cup P$, where $E$ is a set of type $G_{\delta}$ (in natural topology) and $P \in \mathcal{I}$. Here, $E$ is a set of type $F_{\sigma \delta}$ in natural topology and also $F_{\sigma \delta}$ in $\mathcal{T}_{c_{\mu}}$-topology. Simultaneously, $P$ is $\mathcal{T}_{c_{\mu}}$-closed, so is $\mathcal{T}_{c_{\mu}}-F_{\sigma \delta}$ set. Consequently, $A$ is $\mathcal{T}_{c_{\mu}}-F_{\sigma \delta}$ set. 


\section{E. WAGNER-BOJAKOWSKA - W. WILCZYŃSKI - W. WOJDOWSKI}

Obviously, the space $\left(\mathbb{R}, \mathcal{T}_{c_{\mu}}\right)$ is not separable, because all countable sets are closed. Consequently, it is not the second countable (see 4, Corollary 1.3.8., p.44]). It is not the first countable, too (compare [19, Theorem 2.11]).

Additionally, each compact set in this space is finite and $\left(\mathbb{R}, \mathcal{T}_{c_{\mu}}\right)$ is not Lindelöf space (see the proof in [18, Corollary 2]). Furthermore, $\left(\mathbb{R}, \mathcal{T}_{c_{\mu}}\right)$ is a Baire space according to Theorem 7 , and is not regular $\left(T_{3}\right)$ (see the proof in [18, Theorem 6]).

Now, we shall discuss some properties of the continuity of real functions with respect to the $\mathcal{T}_{c_{\mu}}$-topology.

Definition 7. We say that a function $f: \mathbb{R} \rightarrow \mathbb{R}$ is $\mathcal{T}_{c_{\mu}}$-continuous at a point $x_{0}$, if and only if for each $\varepsilon>0$ there exists a set $A_{x_{0}} \in \mathcal{T}_{c_{\mu}}$, such that $x_{0} \in A_{x_{0}}$ and $f\left(A_{x_{0}}\right) \subset\left(f\left(x_{0}\right)-\epsilon, f\left(x_{0}\right)+\epsilon\right)$.

A function $f$ is $\mathcal{T}_{c_{\mu}}$-continuous if it is $\mathcal{T}_{c_{\mu}}$-continuous at each point $x \in \mathbb{R}$.

Using a standard argument, we obtain

THEOREM 9. A function $f: \mathbb{R} \rightarrow \mathbb{R}$ is $\mathcal{T}_{c_{\mu}}$-continuous if and only if $f^{-1}(G) \in \mathcal{T}_{c_{\mu}}$ for each open set $G \subset \mathbb{R}$ (i.e., $f$ is continuous as a function from $\left(\mathbb{R}, \mathcal{T}_{c_{\mu}}\right.$ ) to $(\mathbb{R}, \mathcal{T})$ ).

THEOREM 10. For a function $f: \mathbb{R} \rightarrow \mathbb{R}$, the following conditions are equivalent:

(i) $f$ has the Baire property,

(ii) $f$ is $\mathcal{T}_{c_{\mu}}$-continuous $\mathcal{I}$-almost everywhere on $\mathbb{R}$.

P r o o f. (Compare [13], see also [9].)

(ii) $\Rightarrow$ (i) Suppose $f$ has the Baire property. From [11, Theorem 8.1], there exists a residual set $E \subset \mathbb{R}$ such that the restriction $f \mid E$ is continuous. Then, $f$ is $\mathcal{T}_{c_{\mu}}$-continuous at every point of $E$, i.e., it is $\mathcal{T}_{c_{\mu}}$-continuous $\mathcal{I}$-almost everywhere on $\mathbb{R}$.

(ii) $\Rightarrow$ (i) Suppose now, that $f$ is $\mathcal{T}_{c_{\mu}}$-continuous $\mathcal{I}$-almost everywhere. Let $a, b \in$ $\mathbb{R}, a<b$ and $B=f^{-1}((a, b))$. We shall show that $B$ has the Baire property. Let $C$ be the set of all $\mathcal{T}_{c_{\mu}}$-continuity points of $f$.

We have $B=(B \cap C) \cup(B \backslash C)$ and $B \backslash C \in \mathcal{I}$. The proof is completed by showing that $B \cap C$ has the Baire property. Let $x \in B \cap C$. Then $f(x) \in(a, b)$. From our assumption, there exists a set $A_{x} \in \mathcal{T}_{c_{\mu}}$ such that $x \in A_{x}$ and $f\left(A_{x}\right) \subset(a, b)$. Clearly, $A_{x} \cap C \in \mathcal{T}_{c_{\mu}}, x \in A_{x} \cap C$ and $f\left(A_{x} \cap C\right) \subset(a, b)$. We finally obtain

$$
B \cap C=\bigcup_{x \in B \cap C}\left(A_{x} \cap C\right),
$$

so $B \cap C \in \mathcal{B}$, as $\mathcal{T}_{c_{\mu}} \subset \mathcal{B}$. 


\section{DENSITY TOPOLOGY INVOLVING MICROSCOPIC SETS AND CATEGORY}

THEOREM 11. Every $\mathcal{T}_{c_{\mu}}$-continuous function is approximately continuous, so is a Darboux Baire 1 function.

P r o of. It is a simple consequence of the inclusion

$$
\mathcal{T}_{c_{\mu}} \varsubsetneqq \mathcal{T}_{c}
$$

and the fact that every $\mathcal{T}_{c}$-continuous function is approximately continuous (see [23, Corollary 6]).

THEOREM 12. The family of $\mathcal{T}_{c_{\mu}}$-connected sets coincides with the family of sets connected in the natural topology.

Proof. We can follow here the proof of [19, Theorem 3.7].

THEOREM 13. If a function $f: \mathbb{R} \rightarrow \mathbb{R}$ is $\mathcal{T}_{c_{\mu}}$-continuous, then $f^{-1}((a, b))$ is a countable union of $\mathcal{T}_{c_{\mu}}$-regular open sets for arbitrary interval $(a, b) \subset \mathbb{R}$.

P r o of. Suppose $f: \mathbb{R} \rightarrow \mathbb{R}$ is $\mathcal{T}_{c_{\mu}}$-continuous function and $(a, b) \subset \mathbb{R}$. We have $f^{-1}((a, b))=\Phi_{c_{\mu}}(A) \backslash P$ for some $A \in \mathcal{B}$ and $P \in \mathcal{I}$, and we can assume $P \subset \Phi_{c_{\mu}}(A)$.

Let $x \in \Phi_{c_{\mu}}(A)$. We shall prove that $f(x) \in[a, b]$. Let $\epsilon>0$. Put

$$
D=\Phi_{c_{\mu}}(A) \cap f^{-1}((f(x)-\epsilon, f(x)+\epsilon)) .
$$

Obviously, $x \in D$ and $D \in \mathcal{T}_{c_{\mu}}$, so $D$ is a non-empty set open in $\mathcal{T}_{c_{\mu}}$. Consequently, $D \notin \mathcal{I}$. Hence

$$
D \backslash P=\left(\Phi_{c_{\mu}}(A) \backslash P\right) \cap f^{-1}((f(x)-\epsilon, f(x)+\epsilon)) \neq \emptyset .
$$

It means $f^{-1}((a, b)) \cap f^{-1}((f(x)-\epsilon, f(x)+\epsilon)) \neq \emptyset$, so

$$
(a, b) \cap(f(x)-\epsilon, f(x)+\epsilon) \neq \emptyset .
$$

From the arbitrariness of $\epsilon$, we obtain $f(x) \in[a, b]$. Consequently,

$$
f^{-1}((a, b)) \subset \Phi_{c_{\mu}}(A) \subset f^{-1}([a, b]) .
$$

We can repeat the above considerations for every set of the form $\left(a+\frac{1}{n}, b-\frac{1}{n}\right)$, for $n \in \mathbb{N}$. We have

$$
f^{-1}\left(\left(a+\frac{1}{n}, b-\frac{1}{n}\right)\right)=\Phi_{c_{\mu}}\left(A_{n}\right) \backslash P_{n},
$$

where $A_{n} \in \mathcal{B}, P_{n} \in \mathcal{I}$ and $P_{n} \subset \Phi_{c_{\mu}}\left(A_{n}\right)$ for $n \in \mathbb{N}$. Clearly,

$$
\begin{aligned}
f^{-1}((a, b)) & =\bigcup_{n=1}^{\infty} f^{-1}\left(\left(a+\frac{1}{n}, b-\frac{1}{n}\right)\right) \subset \bigcup_{n=1}^{\infty} \Phi_{c_{\mu}}\left(A_{n}\right), \\
& \subset \bigcup_{n=1}^{\infty} f^{-1}\left(\left[a+\frac{1}{n}, b-\frac{1}{n}\right]\right)=f^{-1}((a, b)) .
\end{aligned}
$$


Thus,

$$
f^{-1}((a, b))=\bigcup_{n=1}^{\infty} \Phi_{c_{\mu}}\left(A_{n}\right)
$$

and according to Remark 3 , the proof is finished.

In [12, O' M a lle y proved that the family of sets

$$
\left\{A \in \mathcal{T}_{d}: m(A)=m(\operatorname{Int}(A))\right\}
$$

forms a topology, called a.e.- topology. He proved that a.e.-topology is completely regular but not normal.

The sets open in a.e.-topology can be characterized as sets of the form $G \cup S$, where $G$ is open in the natural topology $\mathcal{T}$ and $S \subset \Phi_{d}(G)$. Clearly, $S$ can be nowhere dense here and of Lebesgue measure zero.

Simultaneously,

$$
\begin{aligned}
\left\{A \in \mathcal{T}_{d}: A=G \cup S, G \in \mathcal{T}\right. \text { and } & m(S)=0\} \\
= & \left\{B=G \cup S: G \in \mathcal{T} \text { and } S \subset \Phi_{d}(G)\right\} .
\end{aligned}
$$

By analogy, we define $\mathcal{T}_{c_{\mu}}$ - a.e.-topology as a family of sets of the form $G \cup S$, where $G \in \mathcal{T}$ and $S \subset \Phi_{c_{\mu}}(G)$ (compare [20]).

THEOREM 14. If a function $f: \mathbb{R} \rightarrow \mathbb{R}$ is $\mathcal{T}_{c_{\mu}}$-continuous, then $f$ is continuous with respect to the $\mathcal{T}_{c_{\mu}}$ - a.e.-topology.

Pr o of. Let $(a, b) \subset \mathbb{R}$. From the proof of the previous theorem, we have

$$
f^{-1}((a, b))=\bigcup_{n=1}^{\infty} \Phi_{c_{\mu}}\left(A_{n}\right)=\bigcup_{n=1}^{\infty} \Phi_{c_{\mu}}\left(G_{n}\right) \supset \bigcup_{n=1}^{\infty} G_{n},
$$

where $A_{n} \in \mathcal{B}$ and $G_{n}$ is regular open set such that $A_{n} \sim G_{n}$, for $n \in \mathbb{N}$.

Put

$$
G=\bigcup_{n=1}^{\infty} G_{n} \quad \text { and } \quad S=f^{-1}((a, b)) \backslash G .
$$

Obviously, $f^{-1}((a, b))=G \cup S$ and $G \in \mathcal{T}$. On the other hand,

$$
\bigcup_{n=1}^{\infty} \Phi_{c_{\mu}}\left(G_{n}\right) \subset \Phi_{c_{\mu}}(G)
$$

SO

$$
S \subset f^{-1}((a, b))=\bigcup_{n=1}^{\infty} \Phi_{c_{\mu}}\left(G_{n}\right) \subset \Phi_{c_{\mu}}(G) .
$$




\section{DENSITY TOPOLOGY INVOLVING MICROSCOPIC SETS AND CATEGORY}

\section{REFERENCES}

[1] APPELL, J.-D' ANIELLO, E.-VÄTH, M.: Some remarks on small sets, Ricerche Mat. 50 (2001), 255-274.

[2] CIESIELSKI, K.-LARSON, L.-OSTASZEWSKI, K.: I-density continuous functions, Mem. Amer. Math. Soc. 515 (1994), 133 p.

[3] DENJOY, A.: Mémoire sur les dérivés des fonctions continues, J. Math. Pures Appl. 1 (1915), 105-240.

[4] ENGELKING, R.: General Topology. PWN, Warszawa, 1977.

[5] FILIPCZAK, M.-FILIPCZAK, T.: On f-density topologies, Topology Appl. 155 (2008), 1980-1989.

[6] GOFFMAn, C.-NEUGEBAUER, C. J.-NIShIURA, T.: Density topology and approximate continuity, Duke Math. J. 28 (1961), 497-505.

[7] GOFFMAN, C.-WATERMAN, D.: Approximately continuous transformations, Proc. Amer. Math. Soc. 12 (1961), 116-121.

[8] HAUPT, O.-PAUC, H.: La topologie de Denjoy envisagé e comme vraie topologie, C. R. Acad. Sci. Paris 234 (1952), 390-392.

[9] HEJDUK, J.-WIERTELAK, R.: On the abstract density topologies generated by lower and almost lower density operators, in: Traditional and Present-Day Topics in Real Analysis, Łódź University Press, 2013, pp. 431-447.

[10] HORBACZEWSKA, G.-KARAsińSKA, A.-WAGNER-BOJAKOWSKA, E.: Properties of the $\sigma$-ideal of microscopic sets, in: Traditional and Present-Day Topics in Real Analysis, Łódź University Press, 2013, pp. 325-344.

[11] OXTOBY, J. C.: Measure and Category. A Survey of the Analogies Between Topological and Measure Spaces, in: Grad. Texts in Math., Vol. 2, Springer-Verlag, New York, 1980.

[12] O'MALLEY, R. J.: Approximately differentiable functions: The $r$ topology, Pacific J. Math. 72 (1977), 207-222.

[13] POREDA, W.-WAGNER-BOJAKOWSKA, E.-WILCZYŃSKI, W.: A category analogue of the density topology, Fund. Math. 125 (1985), 167-173.

[14] POREDA, W.-WAGNER-BOJAKOWSKA,E.-WILCZYŃSKI, W.: Remarks on I-density and I-approximately continuous functions, Comment. Math. Univ. Carolin. 26 (1985), 553-563.

[15] ROSE, D. A.-JANKOWIĆ, D.-HAMLETT, T. R.: Lower Density Topologies, in: Papers on General Topology and Applications, Proc. of the 7th Summer Conference (S. Andima et al., eds.), Madison, WI, USA, 1991, Ann. N. Y. Acad. Sci., Vol. 704, The New York Academy of Sci., New York, 1993, pp. 309-321.

[16] TALL, F. D.: The density topology, Pacific J. Math. 62 (1976), 275-284.

[17] WAGNER, E.: Sequences of measurable functions, Fund. Math. 112 (1981), 89-102.

[18] WILCZYŃSKI, W.: A generalization of density topology, Real Anal. Exchange 8 (1982-1983), 16-20.

[19] WILCZYŃSKI, W.: Density Topologies, in: Handbook of Measure Theory. Vol I and II. (E. Pap, ed.), North-Holland, Amsterdam, 2002, pp. 675-702.

[20] HEJDUK J.-WILCZYŃSKI, W.-WOJDOWSKI, W.: On semiregularization of the density topologies (submitted). 


\title{
E. WAGNER-BOJAKOWSKA - W. WILCZYŃSKI - W. WOJDOWSKI
}

[21] WILCZYŃSKI, W.-WOJDOWSKI, W.: Density topologies, Problemy Mat. 13 (1992), $45-53$.

[22] WILCZYŃSKI, W.-WOJDOWSKI, W.: A category $\Psi$-density topology, Cent. Eur. J. Math. 9 (2011), 1057-1066.

[23] WOJDOWSKI, W.: Density topology involving measure and category, Demonstratio Math. 22 (1989), 797-812.

[24] WOJDOWSKI, W.: A further generalization of the $T_{\mathcal{A}_{d}}$ density topology, J. Appl. Anal. 19 (2013), 283-304.

Received October 10, 2014

\author{
Elżbieta Wagner-Bojakowska \\ Władysław Wilczyński \\ University of Eódź \\ Faculty of Mathematics and \\ Computer Science \\ Banacha 22 \\ $P L-90-238$ Eódź \\ $P O L A N D$ \\ E-mail: wagner@math.uni.lodz.pl \\ wwil@uni.lodz.pl \\ Wojciech Wojdowski \\ Institute of Mathematics \\ Technical University of Eódź \\ Wólczanska 215 \\ PL-90-924 Eódź \\ POLAND \\ E-mail: wojwoj@gmail.com
}

